\title{
Historia de la Educación a Distancia
}

\author{
(History of Distance Education)
}

\section{Lorenzo García Aretio}

Universidad Nacional de Educación a Distancia (UNED)

(España)

RESUMEN: Los factores más destacados que han provocado el nacimiento y posterior desarrollo de la enseñanza y aprendizaje abiertos y a distancia han sido: los avances sociopolíticos, la necesidad de aprender a lo largo de la vida, la carestía de los sistemas convencionales, los avances en el ámbito de las ciencias de la educación y las transformaciones tecnológicas. La enseñanza a distancia ha evolucionado a través de tres grandes etapas que podemos denominar de la siguiente manera: correspondencia, telecomunicación y telemática. Pues bien, estas grandes fases generacionales, son analizadas en el artículo diferenciando por continentes y países, desde mediados del siglo XIX, hasta nuestra época, señalando los grandes hitos históricos de esta modalidad de enseñar y aprender.

\section{Educación a Distancia - Causas del Nacimiento y Desarrollo - Historia}

ABSTRACT: The most outstanding factors responsible for the birth and subsequent development of open and distance teaching/learning were: socio-political advances, need for all-life-long learning, high costs of conventional systems, advances in the field of education sciences and technological changes. Distance teaching has evolved covering three different stages that could be named: correspondence, telecommunication and telematic. These three great stages are analysed in this article, differentiating by continents and countries, from the second half of XIX century up to date, marking the great historical hits of this modality of teaching/learning.

Distance Education - Birth and Development Causes - History 


\section{INTRODUCCIÓN}

Educar por medios convencionales a todos, atendiendo a satisfacer las múltiples demandas formativas de la sociedad, es hoy prácticamente inviable. En los sistemas educativos de los países desarrollados, los niveles inferiores -enseñanza básica y media- están suficientemente atendidos, pero a partir de la década de los años sesenta, la universidad tradicional, las instituciones de educación de adultos, las empresas dedicadas a la actualización profesional, etc., no logran establecer una infraestructura y organización que pueda atender con agilidad y eficacia a la explosiva demanda de la nueva clientela de esta sociedad industrial.

La masificación de la anquilosada universidad clásica, condujo a un descenso de la calidad de la enseñanza y la imposibilidad de reciclar a todos los trabajadores en servicio hacían un flaco servicio al progreso socioeconómico.

Las aulas convencionales no estaban preparadas para atender esta demanda de formación. Por otra parte no se disponía de recursos económicos suficientes para dotar de personal y medios a las instituciones e instalaciones ya existentes. Incluso los estudiosos del tema abundan en señalar que la solución no iba a venir precisamente a costa de aumentar recursos sin más. Ello sería inútil siempre que no cambiasen las posibilidades del propio sistema educativo.

Esos y otros factores vinieron a impulsar el nacimiento y desarrollo de otras formas de enseñar y aprender en las que no se exigiesen las rigideces espaciotemporales propias de la docencia convencional. Vamos a aproximarnos primeramente a las principales razones que impulsaron el crecimiento de una forma de enseñar/aprender que obviaba la dependencia directa y continua en espacio y tiempo del que aprende con respecto al que enseña.

\section{FACTORES QUE PROPICIAN EL NACIMIENTO Y POSTERIOR DESARROLLO DE LA EDU- CACIÓN A DISTANCIA}

Con la aparición de la escritura se propiciaba el que otros entendiesen un mensaje que una persona distante en el espacio y/o el tiempo, había escrito. Pues a esa revolución de la transmisión de mensajes e ideas a través del medio escrito, siguieron otras que, de manera sintética y cronológica, muestra Wedemeyer (1981) como propiciadoras de formas alternativas de enseñar algo a alguien que está separado en espacio y/o tiempo del hipotético receptor de esa enseñanza. Esta es la secuencia:

- Aparición de la escritura.

- Invención de la imprenta.

D Aparición de la educación por correspondencia.

- Aceptación mayoritaria de las teorías filosóficas democráticas que eliminan los privilegios.

- Uso de los medios de comunicación en beneficio de la educación.

- Expansión de las teorías de enseñanza programada.

Por tanto, si se utilizan racionalmente la escritura, la imprenta, la correspondencia, la telecomunicación y los textos programados, supone Wedemeyer que, puede vencerse plenamente el problema de la distancia o separación entre profesor y alumno.

En efecto, son esos y otros muchos los factores o causas que propiciaron el fenómeno y que se encuentran fuertemente vinculados entre sí. Aunque los motivos del nacimiento y fulgurante desarrollo de la enseñanza/aprendizaje abiertos y a distancia decimos que están muy interrelacionados, trataremos de ofrecer aquellos que nos parecen más significativos. Si bien es verdad, los factores a los que aludimos tuvieron su incidencia, en unos casos en el nacimiento del fenómeno y, en otros, en el desarrollo en determinados países y épocas. (García Aretio, 1986: 25-32):

\subsection{Los avances sociopolíticos}

El aumento de la demanda social de educación generó la masificación de las aulas convencionales, dado que, por una parte surgió la explosión demográfica, y por otra -propiciado por el desarrollo- existía una exigencia y 
presión social justas, de alcanzar todos los niveles de estudios por parte de clases sociales nuevas demandantes del bien de la educación con el consiguiente deterioro de la calidad de ésta.

Los centros convencionales de educación no disponían de infraestructura suficiente para hacer frente a este reto que posibilitase satisfacer los justificados anhelos de democratización de los estudios, reservados hasta entonces a clases más acomodadas, acercándolo a los más, y propiciando así la igualación de oportunidades. Por otra parte, esta creciente masificación de las aulas convencionales desvirtuaba las ventajas de la relación personal profesor-estudiante.

La salida de los bruscos cambios sociales provocados por guerras o revoluciones impulsaba a las sociedades a la búsqueda de vías educativas económicas, accesibles y eficaces para formar a los cuadros y a los profesionales que los nuevos tiempos requerían.

La existencia de desatendidas capas de la población y sectores sociales menos favorecidos en los que había personas que poseían base, motivación y capacidad suficiente como para afrontar con éxito estudios que no tuvieron la oportunidad de cursar y que conformaban un capital humano infrautilizado. Tal sería el caso de:

- los residentes en determinadas zonas geográficas alejadas de los servicios educativos generales convencionales. Adolescentes y jóvenes rurales que aspiran al éxodo hacia las ciudades que ofertan más posibilidades de formación;

- los adultos que por imperativos laborales no pueden acudir a las instituciones clásicas;

- las amas de casa con dificultades para cubrir horarios lectivos ordinarios;

- los hospitalizados o los que sufren algún tipo de minusvalía física que les impide seguir cursos convencionales en el aula;

- los reclusos que disponiendo, generalmente, de mucho tiempo para el estudio no cuentan con la posibilidad de ausentarse del centro penitenciario;

- los emigrantes por causas laborales o los residentes en el extranjero por razones de representación diplomática, etc.;

- los ciudadanos que disponen en sus países, en vías de desarrollo, de pocos centros de enseñanza para atender a todos los que desean acudir a ellos;

- los que tienen demasiados años para acudir a las aulas, pero que no son tan viejos como para no continuar su educación.

Parecía, por tanto, necesario adecuar o crear instituciones educativas que estuviesen en disposición de satisfacer tanta demanda de estos grupos menos favorecidos.

\subsection{La necesidad de aprender a lo largo de la vida}

En las últimas décadas existen una serie de circunstancias en nuestro mundo que nos obligan a un planteamiento radical de los desarrollos educativos. Pretender que la formación de los individuos se circunscriba al periodo escolar en que el alumno es sólo estudiante, es mutilar toda posibilidad de actualización profesional y de progreso social. Existe cada día una mayor solicitud de todo tipo de actividades no regladas de perfeccionamiento profesional, reciclaje..., que impone el progreso de todos los sectores.

Vienen siendo muchos los trabajadores que necesitan cualificarse o recualificarse, así como cuadros y técnicos que han de adaptarse a los nuevos requerimientos productivos, para los que no habían sido preparados.

En esta línea de respuesta se inscribe la educación permanente que viene a considerarse como nueva frontera de la educación y rasgo definitorio del panorama educativo actual. Por ello, los Organismos Internacionales de 
Educación la han convertido en un tema prioritario en sus Recomendaciones porque, en efecto, desde hace años todos quieren aprender a lo largo de toda la vida.

La necesidad de combinar educación y trabajo, con el fin de adaptarse a los constantes cambios culturales, sociales y tecnológicos, sin necesidad de abandonar el puesto laboral pide otra modalidad de formación que no exija la permanencia en el aula.

Las estructuras formales de educación no podían dar respuesta a tantas necesidades de adaptación progresiva a este mundo en cambio y con creciente demanda de educación. Por otra parte, tanto los individuos, desde una aspiración de formación personal, mejoramiento cultural o actualización profesional, como los grupos que tienden a una mejora y bienestar social demandaban nuevas formas de educación que no requiriesen la sistemática asistencia en grupo a un aula de formación. Sería absurdo pensar que la educación continua debe representar una escolarización grupal y en aula, también permanente

\subsection{La carestía de los sistemas convencionales}

La carestía de los sistemas formales de educación era evidente. La educación cara a cara exigía la formación de grupos que precisaban en un determinado espacio y tiempo, de instalaciones y recursos materiales y humanos que si los vinculábamos al todos quieren aprender a lo largo de toda la vida hacían inviable, desde una perspectiva económica, cubrir esa demanda.

Vencer las distancias en países de grandes dimensiones y población escasa y dispersa imponía nuevas propuestas metodológicas que abaratasen la necesidad de que los estudiantes acudieran a las poblaciones que disponían de instituciones presenciales.

El sentido de la rentabilidad de los presupuestos para educación debía llevar a los poderes públicos al convencimiento de que invertir en educación es aumentar el capital humano de una sociedad. Lo que sucede es que a esas inversiones hay que extraerles la máxima rentabilidad en cantidad y calidad, por eso han de buscarse modalidades educativas eficaces y económicas.

Es obvio que mantener de manera exclusiva el sistema escolarizado resultaba utópico, también desde un ángulo económico. No es posible sostener los altos costos de la educación, cuando ésta ha de atender no sólo a la población en edad escolar. Se pedía que parte de las inversiones presupuestarias en educación se reorientasen hacia nuevas formas de enseñar y aprender, sin que ello supusiese el deterioro de la calidad, más bien al contrario, nuevas formas que garantizasen la extensión de una educación de calidad para la mayoría.

Podemos asegurar que hoy son múltiples los estudios que confirman el hecho de la rentabilidad del sistema de educación a distancia, situándolo en un porcentaje muy inferior al de los costes de los sistemas convencionales (Wagner, 1972 y 1977; Carnoy y Levin, 1975, Mace, 1978; Snowden y Daniel, 1980; Rumble, 1986 y 1997; García Aretio, 1987 y Perraton, 1993). El aumento del número de beneficiarios de estos sistemas supone, generalmente, una reducción de los costes generando una economía de escala.

\subsection{Los avances en el ámbito de las ciencias de la educación}

La formación permanente para todos llevaba a la necesidad de flexibilizar las rigideces de la formación convencional a través de la diversificación y ampliación de estudios y cursos reglados y no reglados, ofreciendo otros campos del saber de acuerdo con las necesidades de cada país. Téngase en cuenta, como factor determinante del principio de educación permanente, el del crecimiento exponencial del conocimiento que empuja a tantos adultos a acudir a procesos de aprendizaje de conocimientos y habilidades, necesarios para responder a las demandas de hoy.

Las deficiencias del sistema convencional que además de la imposibilidad estructural de atender a tan amplia demanda, se resistía a una innovación que lo adecuase a los nuevos tiempos y exigencias sociales, llevaba a imaginar nuevas formas de aprendizaje extramural, así como la búsqueda de sistemas educativos innovadores que nos obligasen a un replanteamiento de todas las presunciones convencionales sobre la educación. Pero no olvidemos que la educación convencional es uno de los sistemas más resistentes a los cambios. 
Por otra parte, el sistema convencional está concebido para formar a los estudiantes antes de su participación en las actividades productivas. Esa incapacidad o descuido por la población adulta, activa laboralmente, es un flanco débil en la realización del principio de igualdad de oportunidades.

El notable avance de las Ciencias de la Educación y de la Psicología posibilitaba una planificación cuidadosa de la utilización de recursos y una metodología que, privada de la presencia directa del profesor, potenciaba el trabajo independiente y por ello la personalización del aprendizaje.

Ya se sabe que no es precisa la presencia del profesor para el logro, por parte de la persona adulta, de un aprendizaje personal, reflexivo y significativo. Así, la profundización y perfeccionamiento de los sistemas individualizados de enseñanza, ha logrado la categoría de una modalidad de enseñanza-aprendizaje con rasgos definidos y aplicables a sectores determinados del universo estudiantil.

La sociedad duda que cuanto venía ofreciendo el sistema educativo convencional servía de veras para que el individuo se defendiese y desenvolviese con éxito en la vida. Por ello, la búsqueda de alternativas se imponía.

\subsection{Las transformaciones tecnológicas}

Las transformaciones tecnológicas que permiten reducir la distancia han sido una causa constante del avance insospechado de una enseñanza/aprendizaje no presenciales. Los recursos tecnológicos posibilitan mediante la metodología adecuada suplir, e incluso superar, la educación presencial, con una utilización de los medios de comunicación audiovisual e informáticos integrados dentro de una acción multimedia que posibilita, no sólo la comunicación vertical profesor-estudiante, sino la horizontal entre los propios participantes en los procesos de formación.

El fenómeno de los avances tecnológicos en el campo de la comunicación y de la informática, ha enriquecido los instrumentos de la tecnología educativa con la incorporación de los equipos de grabación, reproducción y transmisión de texto, sonido e imagen. Y no olvidemos que buena parte de estas tecnologías se encuentran hoy en los hogares de multitud de ciudadanos.

Así, los alumnos, a través del estudio independiente, aprenderán al menos de forma tan eficaz como el estudiante de un centro convencional. Más que el método en sí, importa el buen uso que se haga del mismo como muestran numerosas investigaciones.

\section{UN SIGLO Y TRES GENERACIONES DE EDUCACIÓN A DISTANCIA}

Si reflexionamos y comparamos lo que históricamente han supuesto a las diferentes sociedades los avances tecnológicos, con su influencia en el mundo de la educación, deduciremos que no se ha aplicado la tecnología a los procesos formativos con la misma agilidad y eficacia que se ha hecho en otros campos. Por otra parte, no nos constan estudios suficientemente concluyentes referidos a la evaluación del impacto de la tecnología en la educación. En los ámbitos educativos las tecnologías se han utilizado de manera poco sistemática y en no pocos casos ha existido un rechazo claro a la implantación de las mismas (Kerr, 1991).

Los retrasos en la aplicación al mundo educativo de los nuevos artilugios tecnológicos vienen siendo una realidad desde siempre. La comunidad educativa es generalmente conservadora de sus hábitos metodológicos e históricamente le ha costado mucho incorporar los avances tecnológicos. A pesar de ello, y dentro del mundo de la educación, la modalidad a distancia ha sido más receptiva ante estos avances que están revolucionando a la sociedad en todos sus ámbitos.

No cabe duda de que la educación a distancia no es un fenómeno de hoy; en realidad ha sido un modo de enseñar y aprender de millares de personas durante más de cien años. No siempre se aprendió a distancia con el apoyo de los actuales medios electrónicos, sino que esta forma de enseñar y aprender ha evolucionado en el último siglo y medio, a lo largo de tres grandes generaciones de innovación tecnológica que Garrison (1985 y 1989) identifica como correspondencia, telecomunicación y telemática. Aceptamos estas tres etapas, con un matiz en la última de ellas, a las que nosotros les damos la siguiente interpretación.

\subsection{La enseñanza por correspondencia}


Textos muy rudimentarios y poco adecuados para el estudio independiente de los alumnos se usaban casi exclusivamente en esta primera generación, nacida a finales del siglo XIX y principios del XX a lomos del desarrollo de la imprenta y de los servicios postales.

Como bien señala Sauvé (1992), el sistema de comunicación de aquellas instituciones o programas de formación era muy simple, el texto escrito, inicialmente manuscrito, y los servicios nacionales de correos, bastante eficaces, aunque lentos en aquella época, se convertían en los materiales y vías de comunicación de la iniciática educación a distancia. Metodológicamente no existía en aquellos primeros años ninguna especificidad didáctica en este tipo de textos. Se trataba simplemente de reproducir por escrito una clase presencial tradicional.

Poco se tardó en apreciar que así el aprendizaje no era fácil, por lo que se trató de dar una forma más interactiva a ese material escrito mediante el acompañamiento de guías de ayuda al estudio, la introducción sistemática de actividades complementarias a cada lección, así como cuadernos de trabajo, ejercicios y de evaluación, que promoviesen algún tipo de relación del estudiante con la institución, el material y el autor del texto y que facilitasen la aplicación de lo aprendido y guiasen el estudio independiente.

En 1914, l'Ecole Spéciale des Trabaux Publics du Bâtment et de l'Industrie en su publicación l'Enseignement par Correspondence aporta la siguiente definición de enseñanza por correspondencia, muy en consonancia con lo que hemos señalado:

La lección oral del profesor es, con todas las explicaciones y desarrollos que ella comporta, reemplazada por una lección escrita que el alumno no solamente tiene que aprender sino también aplicar.

Ya hacia el final de esta primera etapa se comienza a dibujar la figura del tutor u orientador del alumno que da respuesta por correo a las dudas presentadas por éste, devuelve los trabajos corregidos, anima al estudiante para que no abandone los estudios e incluso mantiene contactos presenciales con él.

Igualmente, para responder mejor a las exigencias de la orientación y guía del alumno se fueron introduciendo paulatinamente en estos estudios de sólo "correspondencia", los aportes de las nacientes tecnologías audiovisuales. Quizás convenga recordar en este punto que allá por 1830 comenzamos a comunicarnos en la distancia a través del telégrafo y sus códigos Morse (1820). En 1876 el escocés A. Graham Bell inventó el teléfono que nos permitió comunicarnos verbalmente a distancia. En 1894 el joven italiano G. Marconi, inventa la radio y en 1901 se realiza la primera comunicación trasatlántica por radio, aunque hasta 1920 no se pone en marcha la primera emisora de radio en Norteamérica. El teletipo (1910) permitía el envío de mensajes escritos a distancia utilizando determinados códigos y en 1923 (Vladimir Zworykin) nace la televisión que, a partir de 1935 efectúa sus primeras emisiones regulares.

Esta etapa ha sido la de mayor duración. Si analizamos hoy multitud de realizaciones de educación a distancia en el mundo, observaremos que muchas de ellas no han traspasado aún esta primera generación, siendo en buena parte de los países la forma más popular de desarrollar esta enseñanza.

\subsection{La enseñanza multimedia}

La enseñanza multimedia a distancia, o en terminología de Garrison, segunda generación se podría situar a finales de los años sesenta (creación de la Open University Británica), viviendo en nuestros días su probable final. Radio y televisión, medios presentes en la mayoría de los hogares, son las insignias de esta etapa. El texto escrito comienza a estar apoyado por otros recursos audiovisuales (audiocasetes, diapositivas, videocasetes, etc.). El teléfono se incorpora a la mayoría de las acciones en este ámbito, para conectar al tutor con los alumnos. En esta segunda generación, al quedar roto el concepto de clase tradicional, las posibilidades de interacción presencial, son escasas.

El diseño, producción y generación de materiales didácticos, dejando en segundo lugar la interacción con los alumnos y de éstos entre sí, son objetivos básicos de estas dos primeras generaciones en enseñanza a distancia.

\subsection{La enseñanza telemática}


La tercera generación, cuyo inicio real podríamos situarlo en la década de los 80, estaría conformada por la educación telemática. La integración de las telecomunicaciones con otros medios educativos, mediante la informática define a esta etapa. Esta tercera generación se apoya en el uso cada vez más generalizado del ordenador personal y de las acciones realizadas en programas flexibles de Enseñanza Asistida por Ordenador (EAO) y de sistemas multimedia. La integración a que aludíamos permite pasar de la concepción clásica de la educación a distancia a una educación centrada en el estudiante.

Se establece algo así como un anillo o malla de comunicaciones al que cada actor del hecho educativo accede desde su propio lugar al resto de sectores con los que debe relacionarse. La inmediatez y la agilidad, la verticalidad y la horizontalidad se hacen presentes en el tráfico de comunicaciones. Garrison contemplaba en esta tercera etapa, básicamente la EAO y las telecomunicaciones florecientes en los años 80. Por eso, entendemos que a esta tercera generación le ha nacido una segunda etapa, la que podríamos centrar en el campus virtual basado en redes de conferencia por ordenador y estaciones de trabajo multimedia, no considerada plenamente por el citado autor.

Habrá de hacerse la aclaración de que las citadas generaciones no se ajustan a períodos cerrados de tiempo ni lugar. Como puede constatarse, y ya decíamos, son aún muchas las realizaciones de enseñanza a distancia que aún no han superado la primera generación. Y, en todo caso, las que se sitúan más claramente en la última etapa continúan utilizando los textos impresos, propios de la primera.

Las diferentes definiciones que sobre educación a distancia han aportado una buena serie de teóricos y estudiosos del campo hemos tenido ocasión de analizarlas en algunos trabajos anteriores ${ }^{1}$.... En la casi totalidad de las mismas se resalta el hecho de que en la educación a distancia han de utilizarse los medios impresos y tecnológicos como puente de unión en el espacio y/o en el tiempo entre profesor y alumno cuando éstos no mantienen una relación cara a cara. En definitiva, se trata de una educación mediada y esa mediación se ha venido realizando con una secuencia ajustada a la evolución de los medios que, durante el siglo y medio real de vida que tiene esta modalidad educativa, podemos resumirla en la siguiente sucesión:

D Texto impreso ordinario.

D Texto impreso con facilitadores para el aprendizaje.

D Tutoría postal.

D Apoyo telefónico.

D Utilización de la radio.

- Aparición de la televisión.

D Apoyo al aprendizaje con audiocasetes.

D Apoyo al aprendizaje con videocasetes.

D Enseñanza asistida por ordenador.

D Audioconferencia.

D Videodisco interactivo.

D Correo electrónico.

D Videoconferencia de sala (grupo).

D WWW (listas, grupos, enseñanza on line...).

D Videoconferencia por Internet.

De la educación por medio impreso y unidireccional, se pasó a la enseñanza por correspondencia y de ésta a la audiovisual. De la enseñanza audiovisual se evolucionó hacia la formación apoyada en la informática, para finalizar con la era de la telemática en la que nos encontramos inmersos. Las tecnologías que se vienen utilizando en esta última era están suponiendo, como decimos, una auténtica revolución en el ámbito de la educación.

\section{DESARROLLO HISTÓRICO DE LA ENSEÑANZA A DISTANCIA}

1 .... Desde 1985 venimos indagando en este tema. Muestra de ello, pueden ser las siguientes publicaciones:

García Aretio, L. (1986). Educación superior a distancia. Análisis de su eficacia. Badajoz: UNED-Mérida.

(1990). "Un concepto integrador de enseñanza a distancia". Villarroel y Pereira. La educación a distancia: desarrollo y apertura. Caracas: ICDE.

(1992). "Le caratteristiche specifiche dell'insegnamento a distanza". Instruzione a Distanza, núm. 6.

(1994). Educación a distancia hoy. Madrid: UNED. 
Los humanos nos comunicábamos a los inicios de nuestra existencia a través de signos y gestos acompañados de sonidos. Posteriormente nos comunicamos a través del lenguaje hablado. Después de esta relación directa se generó la necesidad de comunicaciones en distancias a las que no alcanzaba la voz. Así surgieron las comunicaciones por humo, destellos con espejos, banderas, tambores, etc. Otra fase de la comunicación consistía en la utilización de dibujos mágicos que constituían un ideograma -imagen convencional o símbolo que representa un ser o una idea-, otra fase de esta evolución la representó la escritura jeroglífica de los egipcios palabras representadas por figuras o símbolos- para pasar a la silábica y la alfabética.

Pues bien, existen autores que se remontan hasta las más antiguas civilizaciones para encontrar los orígenes de la enseñanza por correspondencia, germen de la actual enseñanza a distancia. En concreto, Graff (1980) habla de las sumerias y egipcias y en particular señala que la epistolografía griega alcanzó un alto nivel en las cartas de contenido instructivo, gracias a una red de comunicación que se extendía desde Atenas hacia todo el mundo de los antiguos

En realidad, podría afirmarse que este tipo de enseñanza nace con la primera carta escrita por una persona en la que da explicaciones, y ello puede remontase a ejemplos clásicos, como las epístolas de Platón a Dionisios y las cartas de Plinio el Viejo a Plinio el Joven. Las cartas de Séneca (Epistolario a Lucilio) conforman un buen tratado de enseñanza de filosofía estoica. ¿Y qué decir de las cartas de San Pablo a las primitivas comunidades cristianas que tenían un objetivo claramente didáctico?. El humanismo europeo creó el género de exhortationes ad studia literarum que algunas instituciones de enseñanza a distancia de las últimas décadas han utilizado sin, posiblemente, conocer su raíz. Como ejemplos suelen citarse al noble francés Pierre de Maricourt que, en 1269 mediante carta, explica al destinatario amigo los principios del magnetismo. También Newton presentaba epistolarmente al Dr. Bentley argumentos para la existencia de Dios (Popa, 1984).

Igual que Voltaire y Rousseau que utilizaron cartas como vehículo para transmitir sus ideas, son muy reconocidas por su carácter didáctico las Cartas a una princesa alemana, del reconocido matemático Euler, allá por 1770 (Bååt, 1985). Podrían ser estas cartas modelo de muchos materiales impresos de cursos a distancia, en este caso, del área de ciencias.

Sin embargo, no cabe duda que una educación a distancia "por correspondencia", más cercana a lo que hoy entendemos como tal, inicia su desarrollo real al compás de la expansión de los sistemas nacionales de correos, cuyos orígenes se suelen ubicar en el nacimiento en 1680 del penny post, servicio que permitía realizar envíos de cartas o paquetes por el precio de un penique. Un primer dato que nos consta -no tenemos informaciones anteriores- se remonta a cinco décadas después de aquella fecha, a 1728. El 20 de marzo de ese año aparece un anuncio de Caleb Philipps en la Gaceta de Boston ofreciendo material de enseñanza y tutorías por correspondencia (Battenberg, 1971), en los siguientes términos:

\footnotetext{
"Toda persona de la región, deseosa de aprender este arte, puede recibir en su casa varias lecciones semanalmente y ser perfectamente instruida, como las personas que viven en
} Boston” (p. 44)

Si nos basamos en este texto, no parece que se considerase en esta oferta la posibilidad de comunicación bidireccional. Damos un considerable salto en el tiempo, de más de un siglo, para indicar que en 1833 otra reseña similar a la anterior, pero en la que ya podemos reconocer una comunicación de dos vías, fue encontrada en el número 30 del periódico sueco Lunds Weckoblad, en el que se insertaba el siguiente anuncio (Bååth, 1985: 62):

"El abajo firmante, con todo respeto, avisa a aquellas damas y aquellos caballeros de las ciudades vecinas que estudian composición a través del correo, que la dirección para el mes de agosto será Little Grey Friars Street, Lund.

$$
\text { A.J. Meuller" }
$$

Vamos a realizar un breve relato cronológico de los más importantes hitos referidos al nacimiento y desarrollo de este fenómeno educativo y, aunque conscientes de que se nos escaparán algunas referencias de cierto interés, vamos a optar por ofrecerlas mediante una presentación distribuida por grandes áreas geográficas. Finalizaremos con la década de los años 80 , por lo prolijo que resultaría analizar situaciones posteriores.

\subsection{Europa}


En Inglaterra, en 1840, Isaac Pitman programó un sistema de taquigrafía basado en tarjetas e intercambio postal con los alumnos, que tuvo un gran éxito y llega a considerarse por destacados autores como los orígenes reales de la educación a distancia. De hecho había nacido la comunicación didáctica no presencial y bidireccional a través del correo (penny post). La inesperada respuesta de los hipotéticos destinatarios de esa experiencia, llevó a fundar en 1843 la Phonographic Correspondence Society que se encargaba de corregir las tarjetas con los ejercicios de taquigrafía anteriormente aludidos. Este movimiento empezó a interesar en aquellos inicios gracias, tambien, a las conferencias dictadas por el educador escocés James Stuart de la Universidad de Cambridge (Holmberg, 1986).

En 1856 el francés Charles Toussaint, que ya enseñaba su lengua en Alemania, y Gustav Langenscheidt miembro de la Sociedad de Lenguas Modernas de Berlín, impartieron probablemente las primeras clases con material diseñado para el autoestudio fundando el primer instituto para enseñar lenguas extranjeras por correspondencia. Estamos probablemente, por tanto, ante la primera institución verdaderamente de enseñanza por correspondencia, el Institut Toussaint et Langenscheidt (Noffsinger, 1926).

En 1894 el Rustinches Fernlehrinstitut, que ayudó a preparar estudiantes para el examen de acceso a la Universidad (Abitur), fue establecido en Berlín. Una primera experiencia basada en el desarrollo y distribución de materiales autoinstructivos fue el denominado Método Rustin conocido desde 1899 (Delling, 1985).

Los sistemas universitarios a distancia, tan extendidos en la actualidad, quizás tengan su origen en los movimientos de extensión universitaria nacidos allá en la década de los años 60 del siglo XIX, en Estados Unidos. Estos movimientos de extensión, consideraban que el campus es el Estado. En Inglaterra estos movimientos de extensión se iniciaron unos diez años después que en Norteamérica. Unido a ellos creció la idea de que desde la Universidad se podría enseñar a estudiantes que no pudieran acudir regularmente a las aulas universitarias. Así se iniciaba un sistema de estudiantes libres que se registraban en la Universidad, estudiaban independientemente y posteriormente daban examen en dicha Universidad.

Tal sistema fue adoptado de manera muy singular por la Universidad de Londres creada en 1836 (Kaye, 1981). Esta institución tenía por función exclusiva realizar exámenes y conferir grados, sin embargo no impartía docencia. La enseñanza propiamente dicha era impartida por instituciones autorizadas por aquella universidad que tenían autonomía para enseñar pero no para evaluar a los estudiantes (Rumble y Keegan, 1982).

A partir de 1858 este sistema fue mucho más abierto dado que posibilitaba a cualquier persona que superase una prueba de entrada y abonase las respectivas tasas, la admisión a exámenes para la obtención de títulos. Esta circunstancia estimuló el nacimiento de instituciones y colleges dependientes de la Universidad que impartían enseñanza por correspondencia, tales como el Skerry's College, Edinburgh fundado en 1878 que preparaba candidatos para el examen del Servicio Civil; el Foulks Lynch Correspondence Tuition Service en Londres, creado en 1884 que impartía especialización en contabilidad y la University Correspondence College de Cambridge, fundada en 1887 (Holmberg, 1986).

Mientras en 1891 nace en Francia el Centre École Chez Soi, señalaremos que en 1894 el joven británico Joseph William Knipe, preparaba para ejercer el magisterio el Certificated Teachers Examination. Pensando que podía ayudar a otros, publicó un anuncio en The Schoolmaster y así impartió clases por correspondencia a seis estudiantes. Todos ellos superaron los exámenes y al año siguiente se inscribieron treinta alumnos. Así comenzaron modestamente los cursos de Wolsey Hall (Erdos, 1976).

Desarrollos como los enunciados tienen también lugar en los países escandinavos. En Malmoe (Suecia) había, a finales del siglo pasado, una escuela que dirigía Hans Hermods para enseñar idiomas y comercio. Se dió la circunstancia de que uno de los alumnos tuvo que trasladarse a unos $20 \mathrm{kms}$. de Malmoe y Hermods empezó a escribirle cartas. Esa experiencia le llevó a preparar material impreso para enseñar por correspondencia. Parece que Hermods conocía la experiencia de Langenscheidt (Gadden, 1973). En 1898 la casa Hermods ofrece un primer curso por correspondencia de "teneduría de libros" a través del Hermods Korrespondensinstitut que llegó a contar con 150.000 estudiantes matriculados. También la Brevskola-NKI posibilitó el acceso a la enseñanza de nivel secundario a unos pocos miles de estudiantes. En 1914, en Noruega, se funda la Norsk Correspondanseskole como primera institución de educación a distancia en el país, y en Alemania la Fernschule Jena.

A principios de siglo, en 1903, reconocemos en España el nacimiento de la primera experiencia de enseñanza por correspondencia, protagonizada por Julio Cervera Baviera que crea las Escuelas Libres de Ingenieros (Sarramona, 1975). Años más tarde comenzaron sus experiencias algunos centros privados de enseñanza por correspondencia. 
En 1927 la BBC británica inauguró las posibilidades de la radio en el ámbito de la enseñanza, aunque inicialmente sus destinatarios eran los escolares, a los que se les ofrecían programas que complementaban lo aprendido en las aulas.

La ex Unión Soviética tuvo una rica experiencia en un particular sistema de enseñanza por correspondencia, cuyos primeros datos los encontramos en la década de los años 20 del presente siglo (Ljosa, 1991), teniendo un gran desarrollo en las posteriores décadas. Es de destacar que en el curso académico 1940-41 existían en la URSS unos 200.000 alumnos inscritos en estudios de educación superior por correspondencia, poco menos de la tercera parte de todos los estudiantes universitarios de la Unión Soviética en aquel curso. El derecho a la educación a distancia formaba parte de la Constitución de la URSS (Daniel, 1988) que en su artículo 45 señalaba:

"Los ciudadanos soviéticos tienen derecho a la educación. Este derecho está asegurado por la libre provisión de todas las formas de educación... por el desarrollo de cursos de educación a distancia y a tiempo parcial... por la provisión de facilidades para el autoestudio”"

En 1971 eran 600.000 soviéticos los que seguían estudios superiores por correspondencia y Rumble y Keegan (1982) apuntan que en 1979 existían 2.200.000 soviéticos estudiando por este sistema. Hasta hace pocos años el sistema soviético de enseñanza universitaria a distancia se basaba en dos elementos: materiales impresos para el estudio individual de los alumnos y sesiones presenciales regulares (seminarios o tutorías), las cuales podían ser reemplazadas por la correspondencia cuando las distancias fuesen excesivas. Otros países dependientes de la influencia soviética, adoptaron pronto similares propuestas de estudio por correspondencia.

En Francia durante la Segunda Guerra Mundial nació el Centro Nacional de Enseñanza por Correspondencia, posteriormente denominado Centre National d'Enseignement à Distance (CNED) con el fin de mantener en funcionamiento el sistema educativo francés en los niveles primario y secundario atendiendo a los niños huidos de la guerra. Este centro también se apoyaba en emisiones de radio como sistema de mantenimiento y seguimiento de los alumnos (Perraton, 1978).

En todos los ámbitos sociales el final de la Segunda Guerra Mundial supuso muy serias transformaciones socioeconómicas. La necesidad de la población activa laboralmente de responder a los nuevos requerimientos de los mercados de trabajo y a las transformaciones de los sistemas productivos, precisaba de unas propuestas de formación que permitiesen a los trabajadores adquirir esa formación sin necesidad de abandonar el puesto de trabajo. Esta realidad y otras de las señaladas al inicio de este trabajo como factores que impulsaron el desarrollo de esta modalidad de enseñanza/aprendizaje, permitieron la fulgurante expansión de este fenómeno en la segunda mitad del siglo XX, convirtiendo a la educación a distancia en una referencia obligada, cuando en años anteriores a la gran guerra constituía un hecho educativo muy marginal.

En 1947, a través de Radio Sorbonne, se transmitieron clases magistrales en casi todas las materias literarias de la Facultad de Letras y Ciencias Humanas de París. En 1963 se inicia en Francia una enseñanza universitaria por radio en cinco Facultades de Letras (París, Bordeaux, Lille, Nancy, Strasbourg) y en la Facultad de Derecho de París, para los estudiantes de primer curso.

En 1962 se inicia en España una experiencia de Bachillerato radiofónico, un año después se crea el Centro Nacional de Enseñanza Media por Radio y Televisión que sustituyó al Bachillerato radiofónico. En 1968 este Centro se transforma en Instituto Nacional de Enseñanza Media a Distancia (INEMAD).

También en España, en 1963, se constituye Radio ECCA (Emisora Cultural Canaria) que emitió su primera clase radiofónica en 1965. ECCA viene utilizando desde entonces, con algunas variantes, el denominado sistema tridimensional que conjuga la interacción de tres elementos, los esquemas impresos, la clase radiofónica y la tutoría presencial y a distancia.

En esta década surge el afán asociacionista para impulsar la modalidad y defenderla. Así, en 1963 se crea en Bélgica el Consejo Europeo de Educación por Correspondencia (CEC), y en Zurich, en 1968, el Consejo Europeo de Estudios a Domicilio (EHSC), versión europea del National Home Study Council estadounidense. De la fusión de estos dos organismos nació en Dublín, en 1985, la potente Association of European Correspondence Schools (AECS) como asociación internacional para la promoción del conocimiento de la educación a distancia y su defensa, la investigación en ese ámbito, etc. 
El gobierno noruego jugó un destacado papel regulando, en 1948, programas e instituciones de estudios por correspondencia (Moore y Kearsley, 1996). La Norwegian Association for Distance Education - Norway (NADE) fue creada en 1968 como una asociación de escuelas por correspondencia acreditadas. Fue reorganizada en 1984 cuando se abrió a universidades y colegios de educación a distancia. Un consorcio para la educación superior a distancia se creó dentro de NADE en 1986.

En España la hoy consolidada Asociación Nacional de Centros de Enseñanza a Distancia (ANCED), nacida en 1977, destaca entre sus objetivos el de respaldar el logro de un alto nivel de calidad en la enseñanza impartida por los centros a ella vinculados,

El Deutsches Institut für Fernstudienforschung an der Universität Tübingen (DIFF) (Instituto Alemán para la Investigación de la Enseñanza a Distancia) es un instituto para la investigación y desarrollo del aprendizaje abierto a distancia que, aunque tiene estatuto de fundación privada desde 1967, hasta 1977 no fue aceptado como una institución oficial de investigación.

En 1969 se crea la Open University Británica, institución verdaderamente pionera y señera de lo que hoy se entiende como educación superior a distancia. En 1962 Michael Young ya propuso una universidad abierta para preparar a los alumnos externos de la Universidad de Londres. Sin embargo, todas sus energías se volcaron en la fundación del National College Extension y fue Harold Wilson (entonces líder de la oposición) quien en un mitin en Glasgow, en 1963, lanzó la idea de crear una Universidad del Aire que él imaginaba como un consorcio de universidades existentes que usarían las emisiones de radio y televisión y la correspondencia como medios para llevar las enseñanzas a los hogares de los estudiantes adultos. Sin embargo, llegado H. Wilson a Primer Ministro, encargó a su Ministra J. Lee, el desarrollo de la idea que ella modificó en el sentido de crear una universidad autónoma e independiente, igual a las demás y para esto luchó con el fin de vencer todas las resistencias que se le presentaron. Fundada la Open University en 1969, sus primeros 24.000 estudiantes fueron admitidos en 1971.

A partir de esa fecha la expansión de esta modalidad ha sido inusitada. Sólo un año después de que la Open University iniciase sus enseñanzas (1972) se creó la Universidad Nacional de Educación a Distancia (UNED) de España. Posterior a estas dos importantes Universidades unimodales, nacieron en Europa otras instituciones y asociaciones como:

FernUniversität de Hagen, Alemania (1974),

Open Universiteit de Holanda (1982),

National Distance Education Centre de Irlanda (1982),

Swedish Association for Distance Education, Suecia (1984),

Studiecentrum Open Hoger Onderwijs en Bélgica (1987),

Fedération Interuniversitaire de l=Enseignement à Distance de Francia (1987),

Universidade Aberta de Portugal (1988),

The Norwegian Executive Board for Distance Education at University and College Level, Noruega (1990).

La creación de la hoy reconocida European Association of Distance Teaching Universities, EADTU, tuvo lugar en Heerlen (Holanda), en la reunión celebrada el 23 de enero de 1987. Su objetivo fundamental es el del fomento de la educación superior a distancia en Europa.

\subsection{América del Norte}

\subsubsection{Estados Unidos}

Podríamos considerar con Mathieson (1971) que la madre de los estudios por correspondencia en América fue Anna E. Ticknor, hija de un profesor de la Universidad de Harvard, que fundó en Boston, en 1873 la Society for the Promotion of Study at Home (Lambert, 1983).

Un año después inició actividades encaminadas a la realización de un programa por correspondencia apoyado en el material impreso, la metodista Illinois Wesleyan University de Bloomington, institución que consideramos pionera en la enseñanza a distancia de nivel universitario en Estados Unidos (Bittner y Mallory, 1933). En 1883 nacía la Correspondence University de Ithaca, Estado de New York, formada por 32 profesores representantes de prestigiosas universidades convencionales americanas. 
También en 1891, el 14 de julio, el Consejo de Administración de la Universidad de Wisconsin, acordó establecer un curso universitario por correspondencia mediante el que los profesores del Colegio de Agricultura intercambiaron cartas con estudiantes que no podían abandonar su trabajo para volver a las clases en el campus. A principios de siglo, en 1905, en Baltimore se inicia una experiencia de enseñanza elemental a domicilio con la intermediación de padres y gobernantes, destinada a niños, desde la escuela privada Calvert School y la creación de su Departamento de Formación en el Hogar Este ejemplo fue seguido por otros centros en años sucesivos.

Se tiene información de que en 1885 fue establecido un programa por correspondencia en el Chautauqua Institute (Nueva York) gracias a los esfuerzos del profesor de hebreo William. Rainey Harper. Se impartían programas de formación de maestros durante el verano con sesiones presenciales los domingos, que se continuaban por correo. Este centro fue autorizado por el estado de Nueva York para conferir grados oficiales y continuó su actividad hasta 1991 (Vincent, 1886, citado por Garrison y Shale, 1990). Harper consolidaba este método, cuando en 1892 fue nombrado primer Rector de la Universidad de Chicago, que inició sus actividades académicas el 1 de octubre de ese año. En dicha Universidad y de inmediato (octubre de 1892), fundó un departamento o sección de enseñanza por correspondencia, el External Studies Departament. En 1886, ya sentenciaba W. Harper lo siguiente:

\section{Llegará el día en que el volumen de la instrucción recibida por correspondencia será mayor del que es transmitido en las aulas de nuestras escuelas y academias; en que el número de estudiantes por correspondencia sobrepasará a los presenciales.}

Thomas J. Foster, en 1890, muy afectado por los numerosos accidentes mortales que acaecían en las minas, ofreció un curso sobre las medidas de seguridad en las mismas, utilizando como medio de transmisión de los contenidos el periódico de su propiedad Minning Herald de Pennsylvania, del que también era redactor jefe. Un anuncio insertado en dicho periódico en 1890 señalaba las siguientes ventajas del sistema de enseñanza por correspondencia (Moore y Kearsley, 1996, 21):

D Posibilita a los alumnos, desde cualquier distancia, participar en el beneficio de una gran escuela y obtener contacto directo con los escritos de las mayores autoridades en minas.

- El alumno puede comenzar el curso en cualquier momento y puede extender sus estudios por más tiempo y por el mismo coste.

Esta iniciativa se transformó con el paso del tiempo en la denominada desde 1891 International Correspondence Schools de Scranton en Pennsylvania (Daniel y otros, 1982).

En 1906 la Calver School of Baltimore inscribió a cuatro alumnos en sus cursos de estudio en casa, siendo la primera escuela primaria norteamericana en ofrecer cursos por correspondencia (Moore y Kearsley, 1996).

La National University Continuing Education Association (NUCEA) fue organizada en Madison, Wisconsin, en 1915 con el fin de coordinar los cursos por correspondencia de sus escuelas miembro. Los centros de NUCEA ofrecían habitualmente cursos de estudio en casa al nivel de college.

Diferentes centros de estudios por correspondencia florecieron en Estados Unidos, buena parte de ellos fueron miembros del National Home Study Council, una institución federal de carácter privado formada por escuelas de estudios por correspondencia y creada en 1926, con el fin de mejorar los estándares formativos de dichas instituciones. Este organismo, en 1994, ha cambiado su nombre por el de Distance Education and Training Council.

La educación no podía permanecer ajena a los avances tecnológicos, así, en 1917 en la Universidad de Wisconsin se experimentó con una emisora de radio que años después (1922) inició programas educativos, al igual que la University of Minnesota. Aunque parece que la primera licencia de radio educativa fue emitida por el gobierno en 1921 al Latter Day Saints' University of Lake City.

La enseñanza universitaria por correspondencia tuvo un gran protagonismo en Estados Unidos en esta época. Según nos cuentan Bittner y Mallory (1933), en 1930 en este país ofrecían enseñanza por correspondencia 39 universidades

El teléfono, tecnología de utilización básica en cualquier propuesta de enseñanza a distancia que se precie, no permanece al margen de estas nuevas formas de enseñar/aprender, así, en 1939 descubrimos que la 
norteamericana Universidad de Iowa organizó un sistema de enseñanza basado en el teléfono y dirigido a los alumnos que sufrían algún tipo de discapacidad o enfermedad. Así comenzó a utilizarse el teléfono como instrumento de comunicación profesor-alumno.

La irrupción de la televisión no se deja pasar de largo desde las Universidades norteamericanas. En 1956 inicia la emisión de programas educativos por televisión el Chicago TV College cuya influencia se dejó notar pronto en otras universidades del país que no tardaron en crear unidades de enseñanza a distancia, fundamentalmente basadas en la televisión.

Es tradicional en las universidades norteamericanas mantener un serio interés hacia todo lo que sea aprendizaje independiente (open o independent learning) de sus alumnos. Por ello y con el afán de distinguirse de las escuelas de estudio en casa, las universidades norteamericanas decidieron denominar su método como de estudio independiente. Para ello se agruparon en torno a la Independent Study Division of the National University Extension Association. Uno de sus importantes asociados era la Universidad de Wisconsin y sus extended degree programs, así como la Extra-mural Universities de la Carnegie Foundation. En la Universidad de Wisconsin, entre 1964 y 1968, se creó el destacado Proyecto AIM (Articulated Instructional Media) dirigido por Ch. Wedemeyer que trató de integrar a los estudiantes externos a través de la acción de diferentes medios de comunicación, consejo personal o tutoría, grupos de estudio, uso de laboratorios durante el verano, etc. (Moore y Kearsley, 1996). Este proyecto AIM fue la mayor contribución americana a la posterior creación de la Open University Británica.

En 1971 la State University of New York estableció un nuevo college, el Empire State College que nació con el objetivo de ofrecer unos programas de educación alternativa Independent Study Courses dirigido a estudiantes que por diversas circunstancias requerían que sus aprendizajes ocurriesen fuera de las aulas de la Universidad. Esta Universidad cuenta desde 1979 con un relevante Center for Distance Learning.

En similar línea están las experiencias que, desde 1972, realiza la Universidad de Maryland que a través de su University College ha montado una específica Open University Division que ofrece estudios universitarios a distancia.

En 1980 se crea el National University Consortium que produce y emite programas educativos por televisión vía satélite a un buen grupo de instituciones adscritas, aunque no a sus alumnos. Este consorcio en la actualidad, al formar parte de él algunas instituciones canadienses, ha pasado a denominarse International University Consortium for Telecommunications in Learning. Otra asociación importante nacida en la década de los 90 (1992), ha sido la American Associatrion for Collegiate Independent Study (AACIS), nacida más bien para defender los intereses de los profesionales del estudio independiente.

\subsubsection{Canadá}

La enseñanza a distancia de nivel universitario se inició en Canadá en 1889, a través de la Queen's University de Kingston (Ontario). En 1907 la Universidad de Saskatchewan ofrecía a sus alumnos la posibilidad de formarse sin necesidad de acudir a las aulas de clase a través de la Better Farming, los Homemaker short courses y el Canadian Youth Vocational Training Workshops.

En 1919, en Vancouver, se financia con fondos públicos la posibilidad de enseñar por correspondencia a niños alejados de las escuelas. En este país, en la ciudad de Victoria y en 1938, es de destacar la celebración de la Primera Conferencia Internacional sobre la Educación por Correspondencia. Un año antes la Universidad de Ottawa ofrece cursos por correspondencia en las dos lenguas del país, francés e inglés.

Experiencias radiofónicas interesantes surgieron también en Canadá entre los años 30 y 40, tales como la Universidad de San Francisco Javier que promueve el programa Farm Radio Forum, adscrito al Departamento de Extensión de esa Universidad.. A través de Radio Canadá se ofertaban programas de carácter agrícola como el Réveil Rural que facilitó a la escuela de Agricultura emitir programas formativos dirigidos a los agricultores. En la década de los años 40 y 50, Radio Collège utilizó sistemáticamente la radio como apoyo educativo. El Centro de Formación Contínua de la Universidad de la Columbia Británica ofreció a partir de 1950 un programa de cursos por correspondencia desarrollados en directo desde la Facultad de Arte. 
En 1946 se crea en Québec la Oficina de Cursos por Correspondencia, posteriormente denominada Dirección de la Formación a Distancia dependiente del Ministerio de Educación. Esta oficina elaboraba guías para el estudio de los manuales que, también producidos por ella, se destinaban a la enseñanza profesional.

Como instituciones relevantes, más recientemente creadas, podemos destacar: Tele-universite, miembro de la Universite du Quebec, Canadá (1972) y Athabasca University de Alberta, Canadá (1975).

Aunque de ámbito supra canadiense, citamos aquí el nacimiento del más prestigioso organismo asociativo actual en el ámbito de la educación a distancia, se trata del International Council for Distance Education (ICDE) fundado en 1938 en Victoria (Columbia Británica) como el Consejo Internacional para la Educación por Correspondencia (ICCE). Durante sus primeros 20 años de existencia predominaron los miembros norteamericanos interesados en metodología aplicada a centros escolares de enseñanza primaria y secundaria, usando como único medio el impreso. En la Conferencia de Estocolmo de 1965, el Consejo se hizo verdaderamente internacional con 200 delegados asistentes de 27 países. En 1982, en la XII Conferencia Mundial celebrada en Vancouver el nombre ICCE fue cambiado por el actual de ICDE

La Canadian Association for Distance Education (CADE) se constituyó en 1983. Su medio de comunicación más representativo es su revista Journal of Distance Education.

El Consortium International Francophone de Formation à Distance (CIFFAD) fue creado por la Cumbre Francófona en 1987 y en él tiene un papel destacado la Télé-Université de Québec. Se propone establecer contacto con más de un millar de instituciones de 38 países francófonos.

\subsection{Oceanía}

Los países de grandes dimensiones en los que las distancias son enormes, fueron buenos bancos de prueba para el nacimiento de proyectos de enseñanza por correspondencia de niveles primario y secundario financiados por el Estado, al margen de los desarrollos basados fundamentalmente en la iniciativa privada que hemos reseñado hasta ahora. En 1910 W.A. Grundy, Inspector Mayor de Sanidad de Nueva Gales del Sur (Australia), ante las dificultades que tenía para formar a los inspectores de sanidad con destino en zonas rurales, impartió clases por correspondencia a nueve de ellos con cierto éxito. Es un buen ejemplo del origen de la enseñanza profesional por correspondencia (Erdos, 1976).

En Victoria, tambien en 1910, se inicia una experiencia que ha marcado uno de los objetivos básicos de buena parte de la educación a distancia de nuestros días impartida desde diferentes universidades, el de la formación de docentes. La experiencia consistía en que aquellos profesores de nivel primario que desempeñaban su enseñanza en zonas rurales alejadas de los centros superiores de formación, recibían temas por correo, de nivel secundario.

En 1914, el Departamento de educación de Victoria recibió una carta de un colono que vivía a trece kilómetros de la escuela más cercana y preguntaba qué se podía hacer por la educación de sus dos hijos. Cinco estudiantes de magisterio se brindaron para enseñar por correspondencia a esos niños, desde Melbourne. Así se comenzó una experiencia que ayudó a muchos estudiantes aislados de este inmenso país (Rainer, 1949). Este estudio por correspondencia se empezó a denominar en Australia estudios por correspondencia supervisados. Este término daba a entender que los cursos se desarrollaban bajo la guía de un profesor que no necesitaba ser competente en todos los temas de aprendizaje, pero que apoyaba y orientaba a los alumnos siendo el eslabón entre la organización y los estudiantes (Childs, 1963, en Holmberg, 1986).

Tal y como señala Rayner (1949), parece que podemos asegurar que Australia fue el primer país que empleó de forma sistemática y a gran escala la enseñanza por correspondencia en los niveles primario y secundario y para niños que nunca habían ido a la escuela.

El Acta de fundación de la University of Queensland en Brisbane data de 1909 y en ella se autorizaba la impartición de cursos por correspondencia, actividad que se inició en 1911. Quizás podría ser éste, al margen de otras experiencias norteamericanas citadas, el embrión de lo que hoy se ha dado en llamar universidad bimodal (con enseñanza presencial y a distancia). Otros ubican el nacimiento de la bimodalidad universitaria con la University of New England de Armidale (1955), junto a la Universidad de Sudáfrica -a la que posteriormente nos referiremos-. Otras realizaciones de estas caracterìsticas fueron Macquarie University de Sydney (1967); Murdoch University en Perth (1975); en 1978 inició los primeros cursos a distancia la institución mixta Deakin University del Estado de Victoria (Geelong) (Rumble y Keegan, 1982). 
La influencia de la más arriba citada Universidad de Londres, que sólo examinaba a los estudiantes externos sin impartirles docencia, se dejó sentir en Nueva Zelanda. La University of New Zeland en la década de los años 80 del siglo XIX surgió como cuerpo examinador de estudiantes externos (Rumbe y Keegan, 1982). En 1922 en este país comienza sus actividades la New Zeland Correspondence School con la inicial intención de atender a niños aislados, con dificultades para acudir a las aulas convencionales, con algún tipo de minusvalía o enfermedad, así como a adultos deficientemente escolarizados. A partir de 1928 atienden también a alumnos de secundaria.

En la década de los años 40, la radio y el teléfono fueron recursos que empezaban a utilizarse para enseñar a los estudiantes alejados de los centros de enseñanza de la inmensa Australia. A partir de 1950 se utilizaron los servicios radiofónicos del Royal Flying Doctor Service.

En 1951 nacen las Escuelas Australianas del Aire que posibilitan que niños geográficamente aislados participen de la enseñanza diaria con un profesor y otros niños a través de la radio.

En 1963 dos instituciones neozelandesas se unen (Victoria University of Welington y Massey Agrucyultural College) y forman la Massey University Centre for University Extramural Studies de Nueva Zelanda.

En definitiva, Australia y Nueva Zelanda son países donde la educación a distancia está bien establecida y reconocida en todos los niveles del sistema educativo. Como se ha visto, se ha utilizado, desde el principio, en la educación primaria y secundaria, en áreas de población dispersa y para grupos con necesidades especiales. Desde siempre, los padres se han implicado activamente, sobre todo en los niveles primarios (UNESCO, 1998).

Pero Oceanía está formada, además de por Australia y Nueva Zelanda, por otra serie de islas. Quizás convenga destacar la existencia de la singular -parece que no existe otra de estas características- University of the South Pacific que es una Universidad regional creada en 1968 y perteneciente a doce países-islas. Estas islas se extienden a lo largo de 33 millones de Kms. cuadrados de océano -más de tres veces la extensión de Europa- en las que se hablan 200 lenguas diferentes y coexisten 60 culturas. Aunque existen Centros en once de los 12 países, su principal campus está localizado en Laucala Bay en Suva. Los estudiantes de esta Universidad pueden estudiar a tiempo completo, por horas o a distancia, a través de su University Extension.

También en este continentes surgen prestigiosas asociaciones. La Australian and South Pacific External Studies Association (ASPESA) se constituyó en 1972 en Armidale en su Primer Foro Bienal. Los miembros de la asociación proceden fundamentalmente de Fuji, Nueva Guinea Papua, Nueva Zelanda y Australia.

\section{4. África}

La University of South Africa (UNISA), se estableció en 1873 bajo el nombre de Universidad del Cabo de Buena Esperanza como cuerpo examinador -como la Universidad de Londres-, convirtiéndose en Universidad en su pleno sentido en 1916, ya con el nombre actual de Universidad de Sudáfrica con sede en Pretoria (Boucher, 1973) e integrando a aquellas instituciones. En 1946 UNISA marcó un hito en la historia de la educación a distancia universitaria. Oficialmente se empezaron a impartir estudios de nivel superior desde una Universidad convencional, ¿nacimiento de las universidades bimodales?. Hoy es una Universidad de reconocido prestigio internacional que cuenta con unos 140.000 alumnos que siguen estudios a distancia.

La educación a distancia en África también se ha utilizado en la educación no formal y para el desarrollo comunitario en organizaciones nacionales e internacionales. Un ejemplo de ello es la Fundación Panafricana INADES, establecida en 1962 en Costa de Marfil con oficinas nacionales en diez países (UNESCO, 1991).

En 1975 se admiten los primeros alumnos que estudian en la creada Unidad (convertida en 1983 en Correspondence and Open Studies Institute) de educación a distancia de la Universidad de Lagos de Nigeria.

Como en otros continentes, nacen asociaciones. En Africa podemos destacar la African Association for Distance Education (AADE), cuyos inicios se remontan a 1973, nacida en Nairobi como Asociación Africana de Educación por Correspondencia. Su Secretaría Permanente se ubica actualmente en Zimbabwe.

\subsection{Asia}


El Japanese National Public Broadcasting Service (NHK) de Japón iniciaba sus programas escolares de radio en 1935 como complemento y enriquecimiento de la escuela ordinaria. También la televisión se utilizó en Japón con programas educativos desde 1951 (Abe, 1988).

Un primer intento de organización a escala nacional de la enseñanza a distancia en China, data de 1952, bajo las siglas PUC (People's University of China) (Zhou (1992). En 1960 se funda el Beijing Broadcasting and Television, que se cerró como el resto de la educación postsecundaria durante la Revolución Cultural. En este país 1,5 millones de profesores de un total de 5 millones no alcanzaron los niveles de cualificación requeridos en 1989. En consecuencia, se generó un amplio proyecto de adiestramiento de los maestros mediante la enseñanza a distancia, utilizando uno de los dos satélites educativos chinos (UNESCO, 1998).

En la India la enseñanza a distancia data desde mediados del siglo XIX, bajo la forma de enseñanza por correspondencia (Gupta. 1990). En 1962 la Universidad de Delhi abrió un Departamento de Estudios por Correspondencia, como ensayo para atender a los estudiantes que de otro modo no podrían recibir una enseñanza universitaria.

Podemos destacar desarrollos más recientes en Asia, tales como:

Korea Air and Correspondence University, República de Corea (1972) en la actualidad con más 200.000 alumnos,

Free University, Irán (1973), abolida cuatro años después.

Allama Iqbal Open University, Pakistan (1974),

Everyman's University, Israel (1976),

Sukhothai Thammathirat Open University, Thailandia (1978) con más de 200.000 alumnos en nuestros días,

Central Broadcasting and Television University (China TV Univeersity System), China (1978), con más de 500.000 alumnos en la actualidad,

Anadolu University, Turquía (1981) con cerca de 600.000 alumnos matriculados en el umbral del siglo XXI,

Sri Lanka Open University, Sri Lanka (1981),

University of the Air, Japón (1981),

Korea National Open University (1982),

Universitas Terbuka, Indonesia (1983) con 350.000 alumnos en la actualidad,

Open University del estado de Andhara Pradesh de la India (1983),

Indira Gandhi National Open University, India (1985) en la que estudian unos 250.000 alumnos,

National Open University (1986), Taiwan,

Payame Noor University, Irán (1987) hoy con unos 120.000 alumnos.

La idea de concebir la Asian Association of Open Universities (AAOU) nació en 1986 durante el Seminario Regional de Educación a Distancia celebrado en la Sukhothai Thammathirat Open University (STOU) de Bangkok (Thailandia).

\subsection{Iberoamérica}

Méjico es un de los pioneros de la enseñanza a distancia en esta área. En 1947 el Instituto Federal de Capacitación de Magisterio inicia un programa para el perfeccionamiento del profesorado en ejercicio. En 1968 se crearon los Centros de Educación Básica de Adultos (CEBA). En 1971 aparece la Telesecundaria que utiliza la televisión para apoyar la acción de los centros. Otra institución pionera en la aplicación de los sistemas a distancia en México es el Centro para el Estudio de Medios y Procedimientos Avanzados de la Educación (CEMPAE) que, creado en 1971 y extinguido en 1983, inició en 1973 la aplicación de un modelo de Preparatoria Abierta. En 1974 el Instituto Politécnico Nacional inició su Sistema Abierto de enseñanza (SAE) en varias de sus escuelas. También el Colegio de Bachilleres inicia en 1976 su Sistema de Enseñanza Abierto.

Igualmente en Méjico, pero en el ámbito universitario, podemos destacar que en 1972 se iniciaron experiencias de educación a distancia a través del denominado Sistema Universidad Abierta (SUA) de la Universidad Nacional Autonoma de Mexico (UNAM) que hoy ofrece algunos estudios, con validez académica similar a la que la misma Universidad ofrece a través del sistema presencial. Con objeto de racionalizar la multiplicidad de programas se han ido creando en este país diversos organismos gubernamentales. Como ejemplo destacado, 
cabe citar que en 1991 se estableció la Comisión Interinstitucional e Interdisciplinaria de Educación Abierta y a Distancia para coordinar los diversos sistemas y facilitar su interrelación y el planeamiento conjunto.

En Colombia nació un modelo genuinamente latinoamericano con las llamadas escuelas radiofónicas, iniciado por la Acción Cultural Popular, en 1947. A partir de esa experiencia se establecieron programas similares en otros países de la región. Una de las instituciones pioneras de esta área geográfica en la oferta de estudios a distancia fue la Universidad Abierta de la Sabana, con sede central en Bogotá, que impartió los primeros cursos a través de esta modalidad en 1975. Se inició en la misma década otra experiencia de formación a distancia, a través de la televisión; en 1972 la Pontificia Universidad Javeriana emitía por TV el programa Educadores de hombres nuevos. En realidad, en el período 1973-75 fueron ocho las universidades colombianas que establecieron algún programa a distancia (Antioquía, Valle, Javeriana, Santo Tomás...). En 1981 la Universidad de San Buenaventura-Seccional de Cali recogía las iniciales consideraciones del gobierno en cuanto a la solución de problemas de capacitación mediante enseñanzas no convencionales y empezó impartiendo a distancia la Licenciatura en Educación Primaria.

En 1982 el gobierno de Colombia aprueba un Decreto por el cual se reglamenta, dirige e inspecciona la educación abierta y a distancia y se crea el Consejo Nacional de Educación Abierta y a Distancia, así como el Instituto Colombiano para el Fomento de la Educación a Distancia (ICFES), con funciones de promoción, asesoría, capacitación, supervisión y evaluación de los programas a distancia. El Consejo decidió no crear una Universidad unimodal y sí invitar a las instituciones existentes a ofrecer programas a distancia. El gobierno colombiano convirtió a la Unidad Universitaria del Sur (UNISUR) en el centro de innovación en materia de educación a distancia y le asigna funciones de responsabilidad con respecto al desarrollo total del sistema. En el mismo país, Colombia, en 1983 se crean los programas de Educación Abierta y a Distancia de la Universidad Francisco de Paula Santander.

En la República Dominicana los Centros APEC de Educación a Distancia (CENAPEC) se fundan en 1972 con la finalidad de ofrecer programas educativos a bajo costo a dominicanos adultos, marginados socioeconómicamente, mediante el sistema de educación a distancia.

En Ecuador el Instituto Radiofónico Fe y Alegría inició sus programas de enseñanza a distancia en 1972 (en 1976 en Venezuela), dirigidos a adultos con escasa educación. En el mismo país e iniciado en el mismo año, comenzó a funcionar el Sistema de Educación Radiofónico Bicultural Shuar con el objetivo de formar en educación básica a los adultos que hablaban la lengua Shuar. En 1976 comenzó a impartir algunos cursos a distancia la Universidad Técnica Particular de Loja, a través de la denominada Universidad Abierta de Loja.

En Costa Rica, en 1977 la Asamblea Legislativa aprobó la Ley de creación de la Universidad Estatal a Distancia (UNED).

En Venezuela, en 1975 nace la Comisión Organizadora de la Universidad Nacional Abierta (UNA), cuya creación oficial se produjo en 1977. Pocos años antes, en este país, diferentes universidades empezaron a ofrecer algunos estudios a distancia, mediante lo que denominaron Estudios Universitarios Supervisados (EUS).

En Brasil fue fundado en 1939 el Instituto Rádio Monitor y después el Instituto Universal Brasileiro, en 1941. A partir de entonces se sucedieron algunas experiencias de enseñanza a distancia, llevadas a cabo con relativo éxito. El Movimiento de Educaçao de Base (MEB) se preocupaba por alfabetizar y apoyar el aprendizaje de millares de brasileños a través de las escuelas radiofónicas. La situación política generada a partir de 1964, desmanteló este proyecto (Guaranys y Castro, 1979).

La Fundação Educacional Cultura Padre Landell de Moura (FEPLAM) nacida en 1972, tiene su origen en el desarrollo de los movimientos de educación no formal de América Latina. El inicio de FEPLAN fue a través de programas de radio (Colegio do Ar) y de la serie de cursos profesionalizantes (Aprenda pela TV) (Malheiros, 1982). Otra experiencia brasileña digna de reseña es la referida al Serviço Nacional de Aprendizagem Industrial (SENAI) de São Paulo que creó el programa Auto-instrução com Monitoria (AIM) que procuraba impartir mediante la enseñanza a distancia una serie de programaciones autoinstructivas. También la compañía de Petróleos Brasileños (PETROBRAS) experimentó programas para el autoaprendizaje de sus empleados, a partir de 1975 .

Una experiencia brasileña de formación de profesores fue la que desarrolló desde 1973 la Fundação Brasileira de Educação (FUBRAE). Finalmente, referido a Brasil, señalaremos una de las experiencias universitarias de 
educación a distancia, iniciada por la Universidad de Brasilia (UnB) hacia 1976 y que no alcanzó madurez por diferentes circunstancias. Parece que nuevamente se desea retomar este proyecto.

Cuba valoró las necesidades de los trabajadores respecto a la ampliación de las capacidades de matrícula en la educación superior convencional. Para satisfacer esa demanda, el Ministerio de Educación decidió crear la Facultad de Enseñanza Dirigida dentro de la Universidad de La Habana, en 1979.

Experiencias de enseñanza por correspondencia, posteriormente intentos de utilizar la radio y la televisión para realizar estudios, llevaron al Ministerio de educación de Argentina a incluir en el Plan Nacional de Desarrollo y Seguridad para el período 1971/75 distintos cursos a distancia. Así nacieron el plan de formación y actualización de los cuadros de las Fuerzas Armadas y el Plan Nacional de Educación Permanente que inició la Universidad de Buenos Aires. En ese tiempo se crea la Universidad Nacional de Luján que tuvo su importancia en el desarrollo de la modalidad a distancia en Argentina. La Universidad de Belgrano creó en 1983 el Departamento de Educación a Distancia. En 1979 se crea la Asociación Argentina de Educación a Distancia.

Los participantes en el I Symposium Iberoamericano de Rectores de Universidades Abiertas, reunidos en Madrid en octubre de 1980, con el fin de lograr un mayor impulso de la Educación Superior a Distancia en beneficio de los pueblos iberoamericanos, consideraron conveniente crear un mecanismo permanente de información, coordinación y cooperación. Con ese fin nació la Asociación Iberoamericana de Educación Superior a Distancia (AIESAD) con sede en Madrid.

La Comisión Interinstitucional e Interdisciplinaria de Educación Abierta y a Distancia (CIIEAD), se establece en México en 1991 para coordinar los diversos sistemas y facilitar si interrelación y el planeamiento conjunto

En octubre de 1988, en San José de Costa Rica, la Téléuniversité de la Université du Quebec (Canadá) y la Universidad Nacional del Mar de Plata (Argentina) sometieron a consideración de la Junta Directiva de la Organización Universitaria Interamericana (OUI) la idea de formar un consorcio-red de educación a distancia en América (CREAD). La OUI otorgó su acuerdo al proyecto. La constitución definitiva de CREAD tuvo lugar en la Asamblea constituyente de octubre de 1993 celebrada en la Universidad del Estado de Pennsylvania (USA). 


\section{REFERENCIAS BIBLIOGRÁFICAS}

ABE, Y. (1988). Comunications technology. En Sewart, D. y Daniel J.S, Developing Distance Education. Oslo: ICDE, pp. 9-15.

BÅ̊̊, J.A. (1985). Postal Two-way comunication in correspondence education. Malmo: Liberhermods.

BAttenberG, R. W. (1971). The Boston Gazette. March 20, 1728. Epistolodidaktika, 1, 44-45.

BITTNER, W.S. Y MALLORY, H.F. (1933). University Teaching by Mail. New York: Macmillan.

BOUCHER, M. (1973). Spes in arduis - a history of the University of South Africa. Pretoria: University of South Africa.

CARNOY, M. Y LEVIN, H.M. (1975). Evaluation of educational media: some issues. Instructional Science, 4, pp. 385-406.

Daniel, J. S.; Stroud, M.A.; Thompson, J.R. (eds) (1982). Learning at Distance: A World Perspective. Edmonton, Alberta: Athabasca University/ICCE.

DELLING, R.M. (1985). Fernstudium in der Weimarer Republik. ZIFF Papiere 54. Hagen: FernUniersitat.

ERDOS, R.F. (1976). La enseñanza por correspondencia. Madrid: OEI-CEAC-UNESCO.

GADDEN, G. (1973). Hermods 1898-1973. Malmö: Hermods.

GARCíA ARETIO, L. (1986). Educación superior a distancia. Análisis de su eficacia. Badajoz: UNED-Mérida.

GARCÍA ARETIO, L. (1987). Eficacia de la UNED en Extremadura. Badajoz: UNED-Mérida.

GARRISON, D.R. (1985). "Three generations of technological innovation in distance education". Distance Education. N1 6, pp. $235-241$.

GARRISON, D.R. (1989). Understanding distance education. Londres: Routledge.

GARRISON, D.R. Y SHALE, D. (1990). Education at a distance. Malabar, Florida: Robert E. Krieger Publishing Company.

GRAFF, K. (1980). Correspondence instruction in the history of the western world, en Selected Papers on Distance Education, Gesamthockschule, Hagen, ZIFF, Fernuniversität.

GUARANYS, L.R. DOS y CASTRO, C.M. (1979). O ensino por correspondência: uma estratégia de desenvolvimento educacional no Brasil. Brasilia: IPEA.

GUPTA, S.K. (1990). Development of distance education in India: problems, priorities and policies. ICDE Bulletin, vol. 23, pp. 27-33.

HolmberG, B. (1986). Growth and structure of distance education. Beckenham: Croom Helm.

KAYE, A. (1981). Origins and structures. Distance teaching for higher and adult education. Milton: Open University Press.

KERR, S. (1991). Lever and fulcrum: Educational technology in teachers= though and practice. Teachers College Record, Vol. 93

LAmBERT, M. P. (1983). New course planning. The strategy and tactics of developing a home study course. NHSC News. Washington: National Home Study Council.

LJOPSA, E. (1991). Distance education in Europe and models for organizing future cooperation. ICDE Bulletin, vol. 25, pp. 13-24.

MACE, J. (1978). Mythology in the making; is the Open University really cost-effective?. Higher Education, 7, pp. 295-309.

MALHEIROS, R.M. (1982). FEPLAN: uma escola sem paredes. O Comunitário. FEPLAN, $29^{\mathrm{a}}$ ed.

MAthieson, D. E. (1971). Correspondence study: a summary review of the research and development literature. Syracuse, N. Y.: National Home Study Council/ERIC Clearinghouse on adult education.

Moore, M.G. y KeArsley, G. (1996). Distance Education: As systems view. Belmont, CA: Wadswort.

NOFFSINGER, J.S. (1926). Correspondence Scholls, Lyceums, hautauquas. New York: Macmillan.

Perraton, H. (1978). I sat in H. G. Wells chair. Teaching at a Distance, 13, pp. 1-4.

PERRATON, H. (1993). Distance education for teacher training. London: Routledge.

PoPA, D. (1984). Perspectiva europea de la educación superior a distancia. En Boletín AIESAD, 1, 6.

RAYNER, S.A. (Comp.) (1949). Correspondence Education in Australia and New Zealand. Melbourne: University Press.

Rumble, G. (1988). Economics in distance education: time for a change of direction?. En Sewart, D. y Daniel, J.S. Developing Distance Education. Oslo: ICDE, pp. 63-69.

Rumble, G. (1997). The costs and economics of open and distance learning. London: Kogan Page. 
Rumble, G. Y KeEgAN, D. (1982). Distance teaching at university level. En Rumble, G.; Harry, K. Distance Teaching Universities. London: Croom Helm, pp. 15-31.

SARramona, J. (1975). La enseñanza a distancia. Posibilidades y desarrollo actual. Barcelona: CEAC.

SAUVÉ, L. (1992). Origini e sviluppo dell'instruzione a distanza. En Instruzione a Distanza, IV, 2.

SNOWDEN, B.L. Y DANIEL, J.S. (1980). The economics and management of small postsecondary distance education systems. Distance Education, 1, (1), pp. 68-91.

UNESCO (1991). África: A survey of distance education 1991. En New papers on higher education: Studies and research, n1 4. París: UNESCO.

UNESCO (1998). Aprendizaje abierto y a distancia.Perspectiva y consideraciones politicas. Madrid: UNESCO-UNED.

VInCENT, J.H. (1886). The Chautauqua movement. Meadville, Pa.: The Chautauqua Press.

WAGNER, L. (1972). The economics of the Open University. Higher Education, 2, pp. 159-183.

WAGNER, L. (1977). The economics of the Open University revisited. Higher Education, 6, pp. 359-381.

WEDEMEYER, C. A. (1981). Learning at the Back Door. Reflections on Non-Traditional Learning in the Lifespan. Madison: The University of Wisconsin Press.

ZHOU, J. (1992). 40th anniversary of distance education at the People's University of China. ICDE Bulletin, vol. 30, pp. 10-13.

\section{Perfil académico y profesional del autor}

Lorenzo García Aretio es Director del Instituto Universitario de Educación a Distancia de la UNED de España. Es doctor y profesor titular de Teoría de la Educación en la Facultad de Educación. Ha sido también Director Adjunto del Programa de Formación del Profesorado y Coordinador general de Planes de Estudio de la UNED.

Su línea de investigación se viene centrando desde hace bastantes años en todo lo relativo a la enseñanza/aprendizaje abiertos y a distancia. Como autor principal, coordinador o editor, ha publicado 13 libros relativos al tema de educación a distancia y más de 35 artículos en libros y revistas especializadas. Igualmente viene participando en numerosos proyectos financiados por la Unión Europea, siempre relativos al mismo campo.

Lorenzo García Aretio

IUED (UNED)

Ciudad Universitaria, S/N

28040 Madrid

(España)

Tlef.: +34913986681

Fax: +34913986693

Correo-E: ... Lgaretio@edu.uned.es.. 